\title{
Stability for Abstract Evolution Equations
}

\author{
by \\ Patrizia Pucci \\ Università di Perugia \\ Perugia \\ and \\ James Serrin \\ University of Minnesota \\ Minneapolis
}

Dedicated to Carlo Pucci on the occasion of his seventieth birthday

\section{$\S 1$. Introduction}

In a recent paper [6] we studied the question of asymptotic stability for non-autonomous dissipative wave systems. Earlier work in the same direction is due to Marcati $[2,3]$ and Nakao [4], who treated particularly the case of abstract evolution equations. In this note we give a new asymptotic stability theorem which extends the analysis in $[2,3,4]$ by taking into account the techniques introduced in [6].

We focus on abstract equations of the form

$$
\left[P\left(u^{\prime}(t)\right)\right]^{\prime}+A(u(t))+Q\left(t, u^{\prime}(t)\right)+F(u(t))=0,
$$

where $A, F, P$ and $Q$ are nonlinear operators on appropriate Banach spaces. We understand $P$ to be the evolution operator, $A$ as a differential operator of divergence form, $Q$ as a damping term, and $F$ as a restoring force. Concrete examples of (1.1) include the principal case of wave systems, where $P=I, A=-\Delta$, and also, more generally, the $p$-Laplacian, where $A=-\Delta_{p}, p>1$, as well as the polyharmonic operator $A=(-\Delta)^{L}, L \geq 1$.

As an important feature of the present work, we allow the damping $Q=Q(t, v)$ to be strongly non-autonomous in $t$ and nonlinear in $v$. Our main theorem is given in Section 3, while Section 2 is devoted to preliminary results. In particular, in Section 2 we formulate a careful definition of solution of (1.1), which clarifies and generalizes the corresponding definitions in $[2,3,4]$, and moreover resolves the principal difficulty in treating the abstract case, namely that an appropriate energy balance for (1.1) cannot be derived directly, but must instead be inferred from analogy with concrete equations and systems. 


\section{§. Notation and Preliminary Results}

Let $V=\left(V,\|\cdot\|_{V}\right), W=\left(W,\|\cdot\|_{W}\right)$ and $X=\left(X\|\cdot\|_{X}\right)$ be real Banach spaces, $V^{\prime}, W^{\prime}, X^{\prime}$ their dual spaces, and $\langle\cdot, \cdot\rangle_{V},\langle\cdot, \cdot\rangle_{W},\langle\cdot, \cdot\rangle_{X}$ the natural dual pairings. Moreover, we suppose that the spaces $V, W, X$ have a common subspace $G \neq\{0\}$.*

$$
A: W \rightarrow W^{\prime}, \quad F: X \rightarrow X^{\prime} \quad \text { and } \quad P: V \rightarrow V^{\prime}
$$

be functions satisfying the following structural conditions:

(S1) $A, F$ and $P$ are the Fréchet derivatives of real valued $C^{1}$ potentials

$$
\mathcal{A}: W \rightarrow \mathbf{R}, \quad \mathcal{F}: X \rightarrow \mathbf{R}, \quad \mathcal{P}: V \rightarrow \mathbf{R},
$$

respectively, where without loss of generality we assume that $\mathcal{A}(0)=0, \mathcal{F}(0)=0$ and $\mathcal{P}(0)=0$;

(S2) $\langle A(u), u\rangle_{W}+\langle F(u), u\rangle_{X} \geq 0$ for all $u \in G$;

(S3) $\mathcal{P}^{*}(v)=\langle P(v), v\rangle_{V}-\mathcal{P}(v) \geq 0$ in $V$;

(S4) for every $d>0$ the sets

$$
D=\{u \in G: \mathcal{A}(u)+\mathcal{F}(u) \leq d\}, \quad E=\left\{v \in V: \mathcal{P}^{*}(v) \leq d\right\}
$$

are bounded in $X$ and $V$, respectively, and the sets $F(D), P(E)$ are bounded in $X^{\prime}$ and $V^{\prime}$.

It is easily seen that

$$
\mathcal{A}(u)=\int_{0}^{1}\langle A(s u), u\rangle_{W} d s, \quad \mathcal{F}(x)=\int_{0}^{1}\langle F(s x), x\rangle_{X} d s ;
$$

consequently condition (S2) implies that

$$
\mathcal{A}(u)+\mathcal{F}(u) \geq 0 \quad \text { for } u \in G .
$$

Denote the time set $[0, \infty)$ by $J$. Let $S$ be a given subset of $J \times V$ and

$$
Q: S \rightarrow X^{\prime}
$$

a continuous function.

* If $V, W, X$ are themselves subspaces of a common vector space $Z$, then we can take $G=V_{\cap} W_{\cap} X$, this being the maximal subspace contained in all three spaces. As an example, references [3],[4] deal with the special case $W=X \subset V$ (in our notation), for which the appropriate subspace $G$ is simply $X$ itself. 
We consider the abstract evolution equation

$$
\left[P\left(u^{\prime}(t)\right)\right]^{\prime}+A(u(t))+Q\left(t, u^{\prime}(t)\right)+F(u(t))=0, \quad t \in J .
$$

Let $K$ denote the subset of all functions $u: J \rightarrow G$ such that

$$
u \in C^{1}(J \rightarrow V) \cap C(J \rightarrow W) \cap L_{\mathrm{loc}}^{\infty}(J \rightarrow X) .
$$

We say that $u$ is a strong solution of (2.1) if

(a) $u \in K$ and $\left(t, u^{\prime}(t)\right) \in S$ for a.a. $t \in J$;

(b) $u$ verifies (2.1) in the following distribution sense:

$$
\begin{aligned}
\left.\left\langle P\left(u^{\prime}(s)\right), \phi(s)\right\rangle_{V}\right|_{0} ^{t}=\int_{0}^{t}\left\{\left\langle P\left(u^{\prime}(s)\right), \phi^{\prime}(s)\right\rangle_{V}-\langle A(u(s)), \phi(s)\rangle_{W}\right. \\
\left.-\left\langle Q\left(s, u^{\prime}(s)\right), \phi(s)\right\rangle_{X}-\langle F(u(s)), \phi(s)\rangle_{X}\right\} d s
\end{aligned}
$$

for all $\phi \in K$ and $t \in J$.

Note that the first two integrands on the right hand side of (b) are well-defined and integrable on $[0, t], t \in J$. We show at the end of this section, with the help of further structural conditions, that the last two terms also are meaningful.

Let $\mathcal{E}: J \rightarrow \mathbf{R}$ be the total energy of the field $u \in K$, that is

$$
\mathcal{E}(t)=\mathcal{P}^{*}\left(u^{\prime}(t)\right)+\mathcal{A}(u(t))+\mathcal{F}(u(t)) .
$$

(The energy function $\mathcal{E}$, of course, arises naturally for classical conservation laws.) For simplicity in printing, we shall write

$$
\mathcal{H}(t)=-\mathcal{E}(t)
$$

We now postulate the following crucial connection conditions between $Q, u^{\prime}$ and $\mathcal{E}^{\prime}$ (see $[1,6])$ :

(S5) For every strong solution $u$ of (2.1) the corresponding function $\mathcal{H}$ is non-decreasing and absolutely continuous on $J$. Moreover there are exponents $q>m>1$ and a non-negative function $\delta \in L_{\text {loc }}^{1}(J)$ - independent of the solution $u$ - such that

$$
\left\|Q\left(t, u^{\prime}(t)\right)\right\|_{X^{\prime}} \leq[\delta(t)]^{1 / m}\left[\mathcal{H}^{\prime}(t)\right]^{1 / m^{\prime}}+[\delta(t)]^{1 / q}\left[\mathcal{H}^{\prime}(t)\right]^{1 / q^{\prime}} \quad \text { a.e. in } J,
$$

where $m^{\prime}$ and $q^{\prime}$ respectively denote the Hölder conjugates of $m$ and $q$.

(S6) There is a non-negative function $\sigma$ on $J$, with $1 / \sigma \in L_{\mathrm{loc}}^{m-1}(J)$, and a function $\omega=$ $\omega(\tau), \tau \geq 0$, with $\omega(0)=0, \omega$ increasing in $[0,1]$ and $\omega(\tau)=1$ for $\tau \geq 1$, such that

$$
\sigma(t) \omega\left(\left\|u^{\prime}(t)\right\|_{V}\right) \leq \mathcal{H}^{\prime}(t) \quad \text { a.e. in } J
$$


for every solution $u$ of (2.1).

The regularity and monotonicity conditions expressed in (S5) are motivated by the classical conservation balance

$$
\mathcal{H}(t)-\mathcal{H}(0)=\int_{0}^{t}\left\langle Q\left(s, u^{\prime}(s)\right), u^{\prime}(s)\right\rangle d s,
$$

where $\langle\cdot, \cdot\rangle$ denotes an appropriate pairing between $Q$ and $v$. That $Q$ represents a damping is expresed by the requirement that the integrand in (2.4) be non-negative; we are thus led directly to the axiomatic assumption that $\mathcal{H}$ be absolutely continuous and non-decreasing in $J$ along any solution $u$ of $(2.1)$.

In concrete subcases of (2.1), when $V, W$ and $X$ are standard Lebesgue or Sobolev spaces, the pairing $\langle\cdot, \cdot\rangle\rangle$ reduces to an appropriate Lebesgue integration (see [6]).

An abstract case which further clarifies (2.4) occurs when $S=J \times Y$ and $Y$ is a Banach space with continuous inclusions $X \subset Y \subset V$, and $Q: S \rightarrow Y^{\prime}$. Here the pairing $\langle\cdot, \cdot\rangle$ can be specified explicitly as $\langle\cdot, \cdot\rangle_{Y}$. Even more, in this case (2.3) can be derived, up to a multiplicative factor, from the direct conditions

$$
\|Q(t, v)\|_{Y^{\prime}} \leq \delta(t)\left(\|v\|_{Y}^{m-1}+\|v\|_{Y}^{q-1}\right)
$$

and

$$
\|Q(t, v)\|_{Y^{\prime}} \cdot\|v\|_{Y} \leq \gamma\langle Q(t, v), v\rangle_{Y}, \quad \gamma=\text { Const. } \geq 1
$$

(reverse pairing inequality) as one easily checks. It is essentially this case which was discussed in [3,4]; see also the remarks at the end of the paper.

We can now show that the third and fourth integrands in (b) are meaningful for all $t \in J$. First, by (S5), Hölder's inequality, and the fact that $\phi \in L_{\mathrm{loc}}^{\infty}(J \rightarrow X)$ we have

$$
\begin{aligned}
\int_{0}^{t}\left|\left\langle Q\left(s, u^{\prime}(s)\right), \phi(s)\right\rangle_{X}\right| d s \leq \sup _{[0, t]}\|\phi(s)\|_{X}\left[\left(\int_{0}^{t} \delta(s) d s\right)^{1 / m}\left(\int_{0}^{t} \mathcal{H}^{\prime}(s) d s\right)^{1 / m^{\prime}}\right. \\
\left.+\left(\int_{0}^{t} \delta(s) d s\right)^{1 / q}\left(\int_{0}^{t} \mathcal{H}^{\prime}(s) d s\right)^{1 / q^{\prime}}\right]<\infty
\end{aligned}
$$

as required. That the final integral in (b) is well-defined is a consequence of the first part of (2.9) in the following lemma, which will also be useful for the main result of the next section.

LEMMA. Let (S1)-(S5) hold. Then for any strong solution $u$ of (2.1) we have

$$
0 \leq \mathcal{E}(t) \leq \mathcal{E}(0) \quad \text { in } J, \quad \mathcal{E}^{\prime}(t) \leq 0 \quad \text { a.e. in } J
$$

$$
\mathcal{H}^{\prime} \in L^{1}(J)
$$




$$
0 \leq \mathcal{A}(u(t))+\mathcal{F}(u(t)) \leq \mathcal{E}(0), \quad 0 \leq \mathcal{P}^{*}\left(u^{\prime}(t)\right) \leq \mathcal{E}(0) \quad \text { in } J .
$$

Moreover, there exists a constant $C>0$ such that

$$
\|u(t)\|_{X},\|F(u(t))\|_{X^{\prime}} \leq C, \quad\left\|u^{\prime}(t)\right\|_{V},\left\|P\left(u^{\prime}(t)\right)\right\|_{V^{\prime}} \leq C \quad \text { in } J .
$$

PROOF. From (S2)', (S3) and (2.2) we get $\mathcal{E}(t) \geq 0$ in $J$. The condition $(2.6)_{2}$ follows directly from (S5), and in turn also $\mathcal{E}(t) \leq \mathcal{E}(0)$. By (2.6) and the fact that $\mathcal{H} \in A C(J)$, we get (2.7).

Condition (2.8) is a consequence of $(2.6)_{1},(\mathrm{~S} 2)^{\prime}$ and (S3). Relation (2.9) follows immediately from (S4), with $d=\mathcal{E}(0)$.

A final structure condition will be required for our main result (see [6], Lemma 3.4).

(S7) For all $\ell>0$ there exists $\bar{\alpha}=\bar{\alpha}(\ell)>0$ such that $u \in G$ and $\mathcal{A}(u)+\mathcal{F}(u) \geq \ell$ implies $\langle A(u), u\rangle_{W}+\langle F(u), u\rangle_{X} \geq \bar{\alpha}(\ell)$.

\section{$\S 3$. Asymptotic Stability}

We now turn to the main result of the paper.

THEOREM. Let (S1)-(S7) hold. Suppose that there is a non-negative function $k \not \equiv 0$ of class $A C(J)$ such that

$$
\begin{gathered}
\lim _{t \rightarrow \infty} \int_{0}^{t}\left|k^{\prime}(s)\right| d s / \int_{0}^{t} k(s) d s=0, \\
\liminf _{t \rightarrow \infty} \int_{0}^{t}\left(\delta+\sigma^{1-m}\right) k^{m} d s /\left(\int_{0}^{t} k d s\right)^{m}<\infty .
\end{gathered}
$$

Then along any bounded (in $G$ ) strong solution $u$ of (2.1) we have

$$
\lim _{t \rightarrow \infty} \mathcal{E}(t)=0 .
$$

REMARK. It is worth noting that (3.1) implies $k \notin L^{1}(J)$. [Otherwise, we would have $k^{\prime}(t)=0$ on $J$ and $k(t)=$ Const. But then $k$ must be identically zero on $J$, in contradiction with the assumption $k \neq \equiv 0$.] On the other hand, (3.1) obviously holds if $k \notin L^{1}(J)$ and $k^{\prime} \in L^{1}(J)$. The last two conditions were in fact principal requirements in the main stability Theorem 3.1 of [6], so that (3.1) gives a better condition for stability than that result.

Various applications of condition (3.2) are given in Section 5 of [6], to which the reader is referred.

PROOF OF THEOREM. Suppose for contradiction that (3.3) fails along some strong solution $u$ of (2.1). Then by (2.6) there exists $\ell>0$ such that $\mathcal{E}(t) \searrow 2 \ell$ as $t \rightarrow \infty$. 
In what follows, by a standard approximation procedure we can suppose without loss of generality that $k \in C^{1}(J)$. Then since $u \in K$, we also have $\phi=k u \in K$. Put

$$
U=U(t)=\left\langle P\left(u^{\prime}\right), \phi\right\rangle_{V}=k\left\langle P\left(u^{\prime}\right), u\right\rangle_{V} .
$$

By (b) with $\phi=k u \in K$, it follows that

$$
\begin{aligned}
\left.U(s)\right|_{T} ^{t}= & \int_{T}^{t}\left\{k^{\prime}\left\langle P\left(u^{\prime}\right), u\right\rangle_{V}+k\left[\left\langle P\left(u^{\prime}\right), u^{\prime}\right\rangle_{V}+\mathcal{P}^{*}\left(u^{\prime}\right)\right]\right. \\
& \left.-k\left[\mathcal{P}^{*}\left(u^{\prime}\right)+\langle A(u), u\rangle_{W}+\langle F(u), u\rangle_{X}\right]-k\left\langle Q\left(s, u^{\prime}\right), u\right\rangle_{X}\right\} d s \\
= & \int_{T}^{t}\left\{I_{1}+I_{2}+I_{3}+I_{4}\right\} d s,
\end{aligned}
$$

this being valid for all $t \geq T \geq 0$ in $J$.

By (2.9) we have

$$
\int_{T}^{t} I_{1} d s \leq \int_{T}^{t}\left|k^{\prime}(s)\right| \cdot\left\|P\left(u^{\prime}(s)\right)\right\|_{V^{\prime}} \cdot\|u(s)\|_{V} d s \leq C^{2} \int_{T}^{t}\left|k^{\prime}(s)\right| d s .
$$

Next, note that

$$
I_{2} \leq\left[C^{2}+\mathcal{E}(0)\right] k \text { in } J,
$$

by (2.8) and (2.9). Now fix $\vartheta>0$. By (S1) and (S3) the functions $P$ and $\mathcal{P}^{*}$ are continuous on $V$, and $\mathcal{P}^{*}(0)=0$. Hence there exists $\Lambda(\vartheta)>0$ such that

$$
\langle P(v), v\rangle_{V}+\mathcal{P}^{*}(v) \leq \vartheta
$$

for all $v \in V$ with $\|v\|_{V} \leq \Lambda(\vartheta)$. Consequently

$$
I_{2} \leq k \begin{cases}\vartheta & \text { in } J_{1} \\ C+\mathcal{E}(0) & \text { in } J_{2},\end{cases}
$$

where $J_{1}=\left\{t \in J:\left\|u^{\prime}(t)\right\|_{V} \leq \Lambda(\vartheta)\right\}$ and $J_{2}=J \backslash J_{1}$. Now by (S6)

$$
\frac{\mathcal{H}^{\prime}(t)}{\sigma(t)} \geq \omega(\Lambda(\vartheta)) \quad \text { for a.a. } t \in J_{2} .
$$

Thus

$$
I_{2} \leq k\left\{\begin{array}{ll}
\vartheta & \text { in } J_{1} \\
\gamma(\vartheta)\left[\mathcal{H}^{\prime} / \sigma\right]^{1 / m^{\prime}}
\end{array}= \begin{cases}\vartheta k & \text { in } J_{2} \text { (a.e.) } \\
\gamma(\vartheta)\left[\sigma^{1-m} k^{m}\right]^{1 / m}\left[\mathcal{H}^{\prime}\right]^{1 / m^{\prime}}\end{cases}\right.
$$

where

$$
\gamma(\vartheta)=[C+\mathcal{E}(0)] \omega(\Lambda(\vartheta))^{-1 / m^{\prime}}
$$


Therefore, for all $t \geq T \geq 0$,

$$
\int_{T}^{t} I_{2} d s \leq \vartheta \int_{T}^{t} k(s) d s+\gamma(\vartheta) \epsilon(T)\left(\int_{0}^{t} \sigma^{1-m} k^{m} d s\right)^{1 / m}
$$

in view of Hölder's inequality and (2.7), where

$$
\epsilon(T)=\left(\int_{T}^{\infty} \mathcal{H}^{\prime}(s) d s\right)^{1 / m^{\prime}} \rightarrow 0 \quad \text { as } T \rightarrow \infty .
$$

Next, if $\mathcal{P}^{*}\left(u^{\prime}(t)\right) \geq \ell$ at some $t \in J$, then by (S2)

$$
I_{3}(t) \leq-\ell k(t)
$$

On the other hand, if $\mathcal{P}^{*}\left(u^{\prime}(t)\right) \leq \ell$, then

$$
\mathcal{A}(u(t))+\mathcal{F}(u(t)) \geq \ell,
$$

since $\mathcal{E}=\mathcal{P}^{*}\left(u^{\prime}\right)+\mathcal{A}(u)+\mathcal{F}(u) \geq 2 \ell$ on $J$. Hence by $(\mathrm{S} 7)$ and the fact that $u(t) \in G$, we get

$$
\langle A(u(t)), u(t)\rangle_{W}+\langle F(u(t)), u(t)\rangle_{X} \geq \bar{\alpha}(\ell) .
$$

But $\mathcal{P}^{*}\left(u^{\prime}(t)\right) \geq 0$ from (S3), which therefore gives $I_{3}(t) \leq-\bar{\alpha}(\ell) k(t)$. Consequently

$$
I_{3} \leq-\alpha k, \quad \alpha=\alpha(\ell)=\min \{\ell, \bar{\alpha}(\ell)\} .
$$

Let $M(t)=\sup \{k(s): s \in[T, t]\}$. By Young's inequality and the fact that $q>m>1$ we obtain

$$
\left(\int_{T}^{t} \delta k^{q} d s\right)^{1 / q} \leq M(t)^{(q-m) / q}\left(\int_{T}^{t} \delta k^{m} d s\right)^{1 / q} \leq M(t)+\left(\int_{T}^{t} \delta k^{m} d s\right)^{1 / m}
$$

Thus, as in (2.5) with $\phi=u \in K$, we have

$$
\begin{gathered}
\int_{T}^{t} I_{4} d s \leq \sup _{[0, t]}\|u(s)\|_{X}\left[\left(\int_{T}^{t} \delta k^{m} d s\right)^{1 / m} \cdot\left(\int_{T}^{t} \mathcal{H}^{\prime} d s\right)^{1 / m^{\prime}}\right. \\
\left.+\left(\int_{T}^{t} \delta k^{q} d s\right)^{1 / q} \cdot\left(\int_{T}^{t} \mathcal{H}^{\prime} d s\right)^{1 / q^{\prime}}\right] \\
\leq \epsilon_{1}(T)\left[\left(\int_{0}^{t} \delta k^{m} d s\right)^{1 / m}+M(t)\right]
\end{gathered}
$$

where

$$
\epsilon_{1}(T)=C\left[\left(\int_{T}^{\infty} \mathcal{H}^{\prime} d s\right)^{1 / m^{\prime}}+\left(\int_{T}^{\infty} \mathcal{H}^{\prime} d s\right)^{1 / q^{\prime}}\right] \rightarrow 0 \quad \text { as } T \rightarrow \infty
$$


Combining (3.5)-(3.7), (3.9), (3.10) yields, for all $t \geq T \geq 0$,

$$
\begin{aligned}
\left.U(s)\right|_{T} ^{t} \leq C^{2} \int_{T}^{t}\left|k^{\prime}(s)\right| d s & +\theta \int_{T}^{t} k(s) d s+\gamma(\vartheta) \epsilon(T)\left(\int_{0}^{t} \sigma^{1-m} k^{m} d s\right)^{1 / m} \\
& -\alpha \int_{T}^{t} k(s) d s+\epsilon_{1}(T)\left[\left(\int_{0}^{t} \delta k^{m} d s\right)^{1 / m}+M(t)\right] .
\end{aligned}
$$

In view of (3.2) there is a number $B>0$ and a sequence $t_{i} \nearrow \infty$ as $i \rightarrow \infty$ such that, for all $i$,

$$
\int_{0}^{t_{i}}\left(\delta+\sigma^{1-m}\right) k^{m} d s \leq\left(B \int_{0}^{t_{i}} k(s) d s\right)^{m} .
$$

Moreover by (3.1) we can suppose as well that

$$
\int_{0}^{t_{i}}\left|k^{\prime}(s)\right| d s \leq \frac{\alpha}{8 C^{2}} \int_{0}^{t_{i}} k(s) d s .
$$

Now choose $\vartheta=\alpha / 8$ and $T$ so large that

$$
\epsilon(T) \leq \alpha / 8 B \gamma(\vartheta), \quad \epsilon_{1}(T) \leq \min \left\{\alpha / 8 M, C^{2}\right\},
$$

which can be done by (3.8) and (3.11). Then from (3.12)-(3.15) there results, for all $i$ such that $t_{i}>T$,

$$
\begin{aligned}
\left.U(s)\right|_{T} ^{t_{i}} \leq & \frac{\alpha}{8} \int_{0}^{t_{i}} k(s) d s+\frac{\alpha}{8} \int_{T}^{t_{i}} k(s) d s+\frac{\alpha}{8} \int_{0}^{t_{i}} k(s) d s \\
& \quad-\alpha \int_{T}^{t_{i}} k(s) d s+\frac{\alpha}{8} \int_{0}^{t_{i}} k(s) d s+C^{2} M\left(t_{i}\right) \\
= & \frac{7}{8} \alpha \int_{0}^{T} k(s) d s-\frac{\alpha}{2} \int_{0}^{t_{i}} k(s) d s+C^{2} M\left(t_{i}\right) .
\end{aligned}
$$

On the other hand, for $t_{i}>T$,

$$
\left|U\left(t_{i}\right)\right| \leq k\left(t_{i}\right)\left\|P\left(u^{\prime}\left(t_{i}\right)\right)\right\|_{V^{\prime}}\left\|u\left(t_{i}\right)\right\|_{V} \leq C^{2} M\left(t_{i}\right)
$$

and

$$
M\left(t_{i}\right) \leq k(T)+\int_{T}^{t_{i}}\left|k^{\prime}(s)\right| d s \leq k(T)+\frac{\alpha}{8 C^{2}} \int_{0}^{t_{i}} k(s) d s
$$

by (3.14). Hence by (3.16)-(3.18) one easily obtains, for all $i$ sufficiently large,

$$
0 \leq U(T)+2 C^{2} k(T)+\frac{7}{8} \alpha \int_{0}^{T} k(s) d s-\frac{\alpha}{4} \int_{0}^{t_{i}} k(s) d s .
$$

Consequently, since $k$ is non-negative, we find that $k \in L^{1}(J)$.

This provides an immediate contradiction since (3.1) implies that $k \notin L^{1}(J)$, and so completes the proof. 


\section{$\S 4$. Concluding Remarks}

1. The special case $S=J \times Y$ discussed in Section 2 was treated earlier by Nakao [4]. For his main result it was assumed that $V$ is a Hilbert space, that $W=X$, and that $P=I, \mathcal{A}+\mathcal{F}=F_{A}: X \rightarrow \mathbf{R}_{0}^{+}$. In addition, (S5) and (S6) were formulated with the restrictions

$$
\begin{gathered}
0<\text { Const. } \leq \delta(t)=\text { Const. } \sigma(t) \quad \text { in } J, \\
\omega(\tau)=\tau^{m}, \quad m \geq 2
\end{gathered}
$$

and (S7) for the explicit choice $\bar{\alpha}(\ell)=$ Const. $\ell$. Finally, Nakao considered exactly the special function $k \equiv 1$, for which (3.1) and (3.2) are automatically satisfied.

The case when $S=J \times Y$ was also discussed by Marcati [3], but with autonomous damping. The hypotheses in [3] are similar to those in [4], with the exceptions that $P$ need not be the identity, nor $V$ a Hilbert space.

2. If $W$ is continuously imbedded in $X$, slightly generalizing the assumption $W=X$ in [3,4], it is easy to see that the solution space $K$ reduces to $C(J \rightarrow W) \cap C^{1}(J \rightarrow V)$. In this case, from the relation $F \in C\left(X \rightarrow X^{\prime}\right)$ we get

$$
F \circ u \in C\left(J \rightarrow X^{\prime}\right) \text { for all } u \in K,
$$

which is enough to make the final integral in (b) well-defined. Hence the assumption in (S4) that $F(D)$ be bounded in $X^{\prime}$ can be omitted, since its only purpose was to make the relation (b) meaningful. (Of course, when (S4) is thus modified, the consequent implication in (2.9), that $\|F(u(t))\|_{X^{\prime}} \leq C$ along a strong solution of (2.1), must also be dropped.)

3. Condition (S6) need not hold in the entire interval $J$, but in fact can be restricted to a measurable control subset $I \subset J$. The main theorem then needs to be revised only in two places. First, (3.1) should be modified to include the condition

$$
k=0 \quad \text { in } J \backslash I,
$$

and second, (3.2) should be replaced by

$$
\liminf _{t \rightarrow \infty} \int_{[0, t] \cap I}\left(\delta+\sigma^{1-m}\right) k^{m} d s /\left(\int_{0}^{t} k d s\right)^{m}<\infty .
$$

The proofs remain almost the same.

The importance of control subsets in studying asymptotic stability is illustrated in [5], in the context of ordinary differential systems.

Acknowledgment. P. Pucci is a member of Gruppo Nazionale di Analisi Funzionale e sue Applicazioni of the Consiglio Nazionale delle Ricerche. This research has been partly supported by the Italian Ministero dell'Università e della Ricerca Scientifica e Tecnologica. 


\section{REFERENCES}

[1] H.A. Levine \& J. Serrin, A Global Nonexistence Theorem for Quasilinear Evolution Equations with Dissipation, to appear.

[2] P. Marcati, Decay and stability for nonlinear hyperbolic equations, J. Diff. Equations 55 (1984), 30-58.

[3] P. Marcati, Stability for second order abstract evolution equations, Nonlinear Anal. 8 (1984), 237-252.

[4] M. Nakao, Asymptotic stability for some nonlinear evolution equations of second order with unbounded dissipative terms, J. Diff. Equations 30 (1978), 54-63.

[5] P. Pucci \& J. Serrin, Asymptotic stability for intermittently controlled nonlinear oscillators, SIAM J. Math. Anal. 25 (1994), 815-834.

[6] P. Pucci \& J. Serrin, Asymptotic stability for non-autonomous wave systems, to appear. 